\title{
A New Auto-Bäcklund Transformation of the KdV Equation with General Variable Coefficients and Its Application
}

\author{
Chunping Liu \\ Institute of Mathematics, Yangzhou University, Yangzhou 225002, China \\ Correspondence should be addressed to Chunping Liu; liucp@yzu.edu.cn \\ Received 30 April 2014; Accepted 20 June 2014; Published 6 July 2014 \\ Academic Editor: Tiecheng Xia
}

Copyright (C) 2014 Chunping Liu. This is an open access article distributed under the Creative Commons Attribution License, which permits unrestricted use, distribution, and reproduction in any medium, provided the original work is properly cited.

First, by improving some key steps in the homogeneous balance method, a new auto-Bäcklund transformation (BT) to the KdV equation with general variable coefficients is derived. The new auto-BT in this paper does not require the coefficients of the equation to be linearly dependent. Then, based on the new auto-BT in which there is only one quadratic homogeneity equation to be solved, an exact soliton-like solution containing 2-solitary wave is given.

\section{Introduction}

The KdV equation with general variable coefficients reads

$$
u_{t}+f(t) u u_{x}+g(t) u_{x x x}=0,
$$

where $f(t)$ and $g(t)$ are arbitrary analytic functions of $t$, which was originally proposed in [1]. Equation (1) is well known as a model equation describing the propagation of weakly nonlinear and weakly dispersive waves in inhomogeneous media. In recent decades, much progress has been made in the studies of (1) obtaining its auto-BT and exact solutions [2-6]. In [3], by using the truncate Painlevé expansion [7], Hong and Jung obtained an auto-BT which includes 5 Painlevé-Bäcklund equations to be solved. In [4], by using the homogeneous balance principle (HBP) [8-11], $\mathrm{M}$. Wang and Y. Wang derived an auto-BT of (1) as follows:

$$
\begin{gathered}
u(x, t)=12 k(\ln \varphi)_{x x}+V(x, t), \\
\varphi\left(\varphi_{x t}+g \varphi_{x x x x}+V f \varphi_{x x}\right) \\
-\varphi_{x}\left(\varphi_{t}+g \varphi_{x x x}+V f \varphi_{x}\right) \\
+3 g\left(\varphi_{x x}^{2}-\varphi_{x} \varphi_{x x x}\right)=0, \\
V_{t}+f(t) V V_{x}+g(t) V_{x x x}=0, \\
g(t)=k f(t), \quad k=\text { const } \neq 0 .
\end{gathered}
$$

Then, based on auto-BT (2), by using the $\varepsilon$-expansion method, 2-soliton solution of (1) is given and discussed in detail. In [5], Fan obtained an auto-BT of (1) in the following form:

$$
\begin{gathered}
u(x, t)=12 k(\ln \varphi)_{x x}+V(x, t), \\
\varphi_{x} \varphi_{t}+4 g \varphi_{x} \varphi_{x x x}-3 g \varphi_{x x}^{2}+V f \varphi_{x}^{2}=0, \\
\varphi_{x t}+g \varphi_{x x x x}+V f \varphi_{x x}=0, \\
V_{t}+f(t) V V_{x}+g(t) V_{x x x}=0, \\
g(t)=k f(t), \quad k=\text { const } \neq 0 .
\end{gathered}
$$

It is easy to see that auto-BT (3) is a special one of auto-BT (2).

The aim of the present paper is to derive a new autoBT for (1) by improving some key steps in the homogeneous balance method. One can see that the new auto-BT in this paper does not require the coefficients of the equation to be linearly dependent. Then, based on the new auto-BT, an exact soliton-like solution containing 2-solitary wave is given.

\section{Derivation of a New Auto-BT for (1)}

According to the idea of HBP, considering homogeneous balance between $f(t) u u_{x}$ and $g(t) u_{x x x}$ in (1), the first crucial 
step is the assumption that we seek for the auto-BT of (1) in the following form:

$$
\begin{aligned}
u(x, t) & =H(t) \frac{\partial^{2}}{\partial x^{2}}[F(\varphi(x, t))]+v_{0}(x, t) \\
& =H(t)\left[F^{\prime \prime} \varphi_{x}^{2}+F^{\prime} \varphi_{x x}\right]+v_{0}(x, t),
\end{aligned}
$$

where $F=F(\varphi)$ is a function of one argument only, $F^{\prime}=$ $d F / d \varphi, F^{(k)}=d^{k} F / d \varphi^{k}$, the subscripts denote the partial derivatives, $v_{0}(x, t)$ is a given solution of $(1), F(\varphi), \varphi(x, t)$, and $H(t)$ are to be determined later. By using (4), we have

$$
\begin{aligned}
u_{t} & +f(t) u u_{x}+g(t) u_{x x x} \\
& =\left[g(t) H(t) F^{(5)}+f(t) H^{2}(t) F^{\prime \prime} F^{\prime \prime \prime}\right] \varphi_{x}^{5}+\cdots,
\end{aligned}
$$

where the unwritten part in (5) is a polynomial of various partial derivatives of $\varphi(x, t)$, the degree of which is lower than 5. Setting the coefficient of $\varphi_{x}^{5}$ to zero yields an ordinary differential equation for $F(\varphi)$ as follows:

$$
g(t) H(t) F^{(5)}+f(t) H^{2}(t) F^{\prime \prime} F^{\prime \prime \prime}=0,
$$

which has a solution as follows:

$$
F(\varphi)=12 \ln \varphi, \quad H(t)=\frac{g(t)}{f(t)}
$$

thereby

$$
\begin{aligned}
& F^{\prime \prime 2}=-2 F^{(4)}, \quad F^{\prime} F^{\prime \prime \prime}=-4 F^{(4)}, \\
& F^{\prime} F^{\prime \prime}=-6 F^{\prime \prime \prime}, \quad F^{\prime 2}=-12 F^{\prime \prime} .
\end{aligned}
$$

Substituting (4) with (7) into (1) and using (8), we get

$$
\begin{aligned}
u_{t}+f(t) u u_{x}+g(t) u_{x x x} \\
=\left\{H(t) \varphi_{t} \varphi_{x}^{2}+f(t) H^{2}(t)\right. \\
\left.\quad \times\left[4 \varphi_{x}^{2} \varphi_{x x x}-3 \varphi_{x} \varphi_{x x}^{2}\right]+f(t) H(t) v_{0} \varphi_{x}^{3}\right\} F^{\prime \prime \prime} \\
+\left\{2 H(t) \varphi_{x} \varphi_{x t}+H(t) \varphi_{x x} \varphi_{t}+f(t) H^{2}(t)\right. \\
\quad \times\left[5 \varphi_{x} \varphi_{x x x x}-2 \varphi_{x x} \varphi_{x x x}\right]+H^{\prime}(t) \varphi_{x}^{2} \\
\left.+3 f(t) H(t) v_{0} \varphi_{x} \varphi_{x x}+f(t) H(t) v_{0 x} \varphi_{x}^{2}\right\} F^{\prime \prime} \\
+\left\{H(t) \varphi_{x x t}+f(t) H^{2}(t) \varphi_{x x x x x}+H^{\prime}(t) \varphi_{x x}\right. \\
\left.+f(t) H(t)\left[v_{0} \varphi_{x x x}+v_{0 x} \varphi_{x x}\right]\right\} F^{\prime} \\
+v_{0 t}+f(t) v_{0} v_{0 x}+g(t) v_{0 x x x}=0 .
\end{aligned}
$$

Decompose the coefficient of $F^{\prime \prime \prime}$ as

$$
\begin{aligned}
& \left\{H(t) \varphi_{t} \varphi_{x}+f(t) H^{2}(t)\left[4 \varphi_{x} \varphi_{x x x}-3 \varphi_{x x}^{2}\right]\right. \\
& \left.\quad+f(t) H(t) v_{0} \varphi_{x}^{2}\right\} \varphi_{x} .
\end{aligned}
$$

Denote

$$
\begin{aligned}
A= & H(t) \varphi_{t} \varphi_{x}+f(t) H^{2}(t)\left[4 \varphi_{x} \varphi_{x x x}-3 \varphi_{x x}^{2}\right] \\
& +f(t) H(t) v_{0} \varphi_{x}^{2} .
\end{aligned}
$$

Integrate the coefficient of $F^{\prime}$ with respect to $x$ once and let the constant of integration be zero. Denote

$$
\begin{aligned}
B= & H(t) \varphi_{x t}+f(t) H^{2}(t) \varphi_{x x x x}+H^{\prime}(t) \varphi_{x} \\
& +f(t) H(t) v_{0} \varphi_{x x} .
\end{aligned}
$$

Rewriting the coefficient of $F^{\prime \prime}$, we have

$$
\text { Coeff. of } F^{\prime \prime}=A_{x}+B \varphi_{x} \text {. }
$$

Substituting (11), (12), and (13) into (9) gives

$$
A \varphi_{x} F^{\prime \prime \prime}+\left(A_{x}+B \varphi_{x}\right) F^{\prime \prime}+B_{x} F^{\prime}=0
$$

that is,

$$
\frac{24 A \varphi_{x}}{\varphi^{3}}-\frac{12\left(A_{x}+B \varphi_{x}\right)}{\varphi^{2}}+\frac{12 B_{x}}{\varphi}=0 .
$$

By direct calculating, we can write (15) as follows:

$$
12\left(\frac{B \varphi-A}{\varphi^{2}}\right)_{x}=0 .
$$

Note the following:

$$
\begin{aligned}
B \varphi-A=\{ & H(t) \varphi_{x t}+f(t) H^{2}(t) \varphi_{x x x x} \\
& \left.+H^{\prime}(t) \varphi_{x}+f(t) H(t) v_{0} \varphi_{x x}\right\} \varphi \\
- & \left\{H(t) \varphi_{t} \varphi_{x}+f(t) H^{2}(t)\right. \\
& \left.\times\left[4 \varphi_{x} \varphi_{x x x}-3 \varphi_{x x}^{2}\right]+f(t) H(t) v_{0} \varphi_{x}^{2}\right\} ;
\end{aligned}
$$

thus we have a new auto-Bäcklund transformation of (1) as follows:

$$
\begin{gathered}
u(x, t)=12 H(t) \frac{\partial^{2}}{\partial x^{2}}[\ln \varphi(x, t)]+v_{0}(x, t), \\
H(t)=\frac{g(t)}{f(t)} \\
\left\{H(t) \varphi_{x t}+f(t) H^{2}(t) \varphi_{x x x x}\right. \\
\left.+H^{\prime}(t) \varphi_{x}+f(t) H(t) v_{0} \varphi_{x x}\right\} \varphi \\
-\left\{H(t) \varphi_{t} \varphi_{x}+f(t) H^{2}(t)\left[4 \varphi_{x} \varphi_{x x x}-3 \varphi_{x x}^{2}\right]\right. \\
\left.+f(t) H(t) v_{0} \varphi_{x}^{2}\right\}=0 .
\end{gathered}
$$

The meaning of the new auto-BT is that if $v_{0}=v_{0}(x, t)$ is a given solution of (1) and $\varphi=\varphi(x, t)$ is a solution of (19), then expression (18) is another solution of (1).

The result shows that the new auto-BT does not require the coefficients of the equation to be linearly dependent; in fact, there are no constraints between $f(t)$ and $g(t)$. 


\section{Exact Soliton-Like Solutions of (1)}

Now, we use the auto-BT that consisted of (18) and (19) to find soliton-like solutions of (1).

First, notice that, under the condition $H(t)=\widehat{H}(t)=$ $a_{0} \int^{t} f(s) d s+b_{0},\left(a_{0}^{2}+b_{0}^{2} \neq 0\right)$, (1) admits a special solution as follows:

$$
v(x, t)=\widehat{v}_{0}=\frac{a_{0} x+c_{0}}{a_{0} \int^{t} f(s) d s+b_{0}},
$$

where $a_{0}, b_{0}$, and $c_{0}$ are arbitrary constants. After a direct calculation, we find the following:

$$
\begin{aligned}
\widehat{H}^{\prime}(t) \varphi_{x}+f(t) \widehat{H}(t) \widehat{v}_{0} \varphi_{x x} & =\left[\left(a_{0} x+c_{0}\right) f(t) \varphi_{x}\right]_{x} \\
& =\left[f(t) \widehat{H}(t) \widehat{v}_{0} \varphi_{x}\right]_{x} .
\end{aligned}
$$

Then, (19) becomes the following form:

$$
\begin{aligned}
\varphi\left\{\widehat{H}(t) \varphi_{t}+f(t) \widehat{H}^{2}(t) \varphi_{x x x}+\left(a_{0} x+c_{0}\right) f(t) \varphi_{x}\right\}_{x} \\
-\varphi_{x}\left\{\widehat{H}(t) \varphi_{t}+f(t) \widehat{H}^{2}(t) \varphi_{x x x}+\left(a_{0} x+c_{0}\right) f(t) \varphi_{x}\right\} \\
\quad-3 f(t) \widehat{H}^{2}(t)\left[\varphi_{x} \varphi_{x x x}-\varphi_{x x}^{2}\right]=0 .
\end{aligned}
$$

Denote

$$
\begin{gathered}
P=\widehat{H}(t) \partial_{t}+f(t) \widehat{H}^{2}(t) \partial_{x}^{3}+\left(a_{0} x+c_{0}\right) f(t) \partial_{x}, \\
P[\varphi]=\widehat{H}(t) \varphi_{t}+f(t) \widehat{H}^{2}(t) \varphi_{x x x}+\left(a_{0} x+c_{0}\right) f(t) \varphi_{x} ;
\end{gathered}
$$

thus (22) can be rewritten as follows:

$$
\varphi\{P[\varphi]\}_{x}-\varphi_{x} P[\varphi]-3 f(t) \widehat{H}^{2}(t)\left[\varphi_{x} \varphi_{x x x}-\varphi_{x x}^{2}\right]=0 .
$$

It is not difficult to find that linear equation $P[\varphi]=0$ admits an exponential solution as follows:

$$
\varphi(x, t)=1+\exp \eta, \quad \eta=p(t) x+q(t)+\eta_{0},
$$

where

$$
\begin{gathered}
p(t)=\frac{\beta}{a_{0} \int^{t} f(s) d s+b_{0}}, \\
q(t)=-\int^{t} \frac{\left[\beta^{3}+c_{0} \beta\right] f(\tau)}{\left[a_{0} \int^{\tau} f(s) d s+b_{0}\right]^{2}} d \tau,
\end{gathered}
$$

and $\eta_{0}$ and $\beta$ are arbitrary constants. In fact, substituting (25) into $P[\varphi]=0$ gives

$$
\begin{aligned}
& \left\{\widehat{H}(t)\left[p^{\prime}(t) x+q^{\prime}(t)\right]+f(t) \widehat{H}^{2}(t) p^{3}(t)\right. \\
& \left.+\left(a_{0} x+c_{0}\right) f(t) p(t)\right\} \exp \eta=0 .
\end{aligned}
$$

Let

$$
\begin{gathered}
\widehat{H}(t) p^{\prime}(t)+a_{0} f(t) p(t)=0, \\
\widehat{H}(t) q^{\prime}(t)+f(t) \widehat{H}^{2}(t) p^{3}(t)+c_{0} f(t) p(t)=0,
\end{gathered}
$$

and solving (28) yields (26). In addition, noting that the third part of (24) vanishes if it is substituted with (25), we immediately find that (25) with (26) is a solution of (24). Substituting (25) with (26) and $\widehat{v}_{0}$ into (18) yields a new exact solution containing a single solitary wave of (1) as

$$
\begin{aligned}
u(x, t)= & \left(\frac{a_{0} x+c_{0}}{a_{0} \int^{t} f(s) d s+b_{0}}\right)+\left(\frac{3 \beta^{2}}{a_{0} \int^{t} f(s) d s+b_{0}}\right) \\
& \times \operatorname{sech}^{2} \frac{1}{2}\left[p(t) x+q(t)+\eta_{0}\right]
\end{aligned}
$$

where $p(t)$ and $q(t)$ are expressed by (26).

From (29), it is seen that the amplitude of single solitary wave is $3 \beta^{2}\left[a_{0} \int^{t} f(s) d s+b_{0}\right]^{-1}$; and the propagating speed of single solitary wave is given by

$$
\frac{d x}{d t}=\frac{\beta^{2} f(t)+\left(a_{0} x+c_{0}\right) f(t)}{a_{0} \int^{t} f(s) d s+b_{0}}
$$

which depends upon not only time $t$ but also the spatial variable $x$.

In order to obtain more general solutions of (1), according to auto-BT that consisted of (18) and (19), we need to seek more general solutions of homogeneity equation (24). Take

$$
\begin{gathered}
\stackrel{\star}{\varphi}(x, t)=1+\exp \eta_{1}+\exp \eta_{2}+a_{12} \exp \left(\eta_{1}+\eta_{2}\right), \\
\eta_{i}=p_{i}(t) x+q_{i}(t)+\eta_{i 0}, \quad(i=1,2),
\end{gathered}
$$

where

$$
\begin{gathered}
p_{i}(t)=\frac{\beta_{i}}{a_{0} \int^{t} f(s) d s+b_{0}}, \\
q_{i}(t)=-\int^{t} \frac{\left[\beta_{i}^{3}+c_{0} \beta_{i}\right] f(\tau)}{\left[a_{0} \int^{\tau} f(s) d s+b_{0}\right]^{2}} d \tau,
\end{gathered}
$$

$\eta_{i 0}$ and $\beta_{i}(i=1,2)$ are arbitrary constants, and $a_{12}$ is an undetermined constant. Because $P$ is a linear operator and $P\left[\exp \eta_{i}\right]=0,(i=1,2)$, 


$$
\begin{aligned}
P[\stackrel{\star}{\varphi}]= & P\left[1+\exp \eta_{1}+\exp \eta_{2}+a_{12} \exp \left(\eta_{1}+\eta_{2}\right)\right] \\
= & a_{12} P\left[\exp \left(\eta_{1}+\eta_{2}\right)\right] \\
= & a_{12}\left\{\widehat{H}(t)\left\{\left[p_{1}^{\prime}(t)+p_{2}^{\prime}(t)\right] x+q_{1}^{\prime}(t)+q_{2}^{\prime}(t)\right\}\right. \\
& +f(t) \widehat{H}^{2}(t)\left[p_{1}(t)+p_{2}(t)\right]^{3} \\
& \left.+\left(a_{0} x+c_{0}\right) f(t)\left[p_{1}(t)+p_{2}(t)\right]\right\} \\
& \times \exp \left(\eta_{1}+\eta_{2}\right) \\
= & 3 a_{12} f(t) \widehat{H}^{2}(t) p_{1}(t) p_{2}(t) \\
& \times\left[p_{1}(t)+p_{2}(t)\right] \exp \left(\eta_{1}+\eta_{2}\right) .
\end{aligned}
$$

Thus,

$$
\begin{aligned}
\{P[\stackrel{\star}{\varphi}]\}_{x}= & 3 a_{12} f(t) \widehat{H}^{2}(t) p_{1}(t) p_{2}(t) \\
& \times\left[p_{1}(t)+p_{2}(t)\right]^{2} \exp \left(\eta_{1}+\eta_{2}\right) .
\end{aligned}
$$

Substituting (31) and (32) into the first two parts on the lefthand side of (24), we get

$$
\begin{aligned}
\stackrel{\star}{\varphi}\{P[\stackrel{\star}{\varphi}]\}_{x}-\stackrel{\star}{\varphi}_{x} P[\stackrel{\star}{\varphi}] \\
=3 a_{12} f(t) \widehat{H}^{2}(t) p_{1}(t) p_{2}(t) \\
\quad \times\left[p_{1}(t)+p_{2}(t)\right]^{2} \exp \left(\eta_{1}+\eta_{2}\right) \\
+3 a_{12} f(t) \widehat{H}^{2}(t) p_{1}^{2}(t) p_{2}(t) \\
\quad \times\left[p_{1}(t)+p_{2}(t)\right] \exp \left(\eta_{1}+2 \eta_{2}\right) \\
+3 a_{12} f(t) \widehat{H}^{2}(t) p_{1}(t) p_{2}^{2}(t) \\
\quad \times\left[p_{1}(t)+p_{2}(t)\right] \exp \left(2 \eta_{1}+\eta_{2}\right)
\end{aligned}
$$

In addition, by direct calculating, we obtain

$$
\begin{aligned}
& \stackrel{\star}{\varphi_{x}} \stackrel{\star}{\varphi}_{x x x}-\left[\stackrel{\star}{\varphi}_{x x}\right]^{2} \\
& =p_{1}(t) p_{2}(t)\left[p_{1}(t)-p_{2}(t)\right]^{2} \exp \left(\eta_{1}+\eta_{2}\right) \\
& \quad+a_{12} p_{1}^{2}(t) p_{2}(t)\left[p_{1}(t)+p_{2}(t)\right] \exp \left(\eta_{1}+2 \eta_{2}\right) \\
& \quad+a_{12} p_{1}(t) p_{2}^{2}(t)\left[p_{1}(t)+p_{2}(t)\right] \exp \left(2 \eta_{1}+\eta_{2}\right) .
\end{aligned}
$$

Substituting (35) and (36) into (24) gives

$$
\begin{aligned}
3 f(t) & \widehat{H}^{2}(t) p_{1}(t) p_{2}(t) \\
\times & \left\{a_{12}\left[p_{1}(t)+p_{2}(t)\right]^{2}\right. \\
& \left.\quad-\left[p_{1}(t)-p_{2}(t)\right]^{2}\right\} \exp \left(\eta_{1}+\eta_{2}\right)=0 .
\end{aligned}
$$

If

$$
a_{12}=\frac{\left[p_{1}(t)-p_{2}(t)\right]^{2}}{\left[p_{1}(t)+p_{2}(t)\right]^{2}}=\left(\frac{\beta_{1}-\beta_{2}}{\beta_{1}+\beta_{2}}\right)^{2},
$$

then $\stackrel{\star}{\varphi}(x, t)$ is a solution of (24). Therefore, a new exact soliton-like solution containing 2-solitary wave for (1) is obtained from

$$
\begin{aligned}
u(x, t)= & \left(\frac{a_{0} x+c_{0}}{a_{0} \int^{t} f(s) d s+b_{0}}\right) \\
& +12\left[a_{0} \int^{t} f(s) d s+b_{0}\right] \frac{\partial^{2}}{\partial x^{2}}[\ln \stackrel{\star}{\varphi}(x, t)],
\end{aligned}
$$

where

$$
\begin{gathered}
\stackrel{\star}{\varphi}(x, t)=1+\exp \eta_{1}+\exp \eta_{2}+\left(\frac{\beta_{1}-\beta_{2}}{\beta_{1}+\beta_{2}}\right)^{2} \exp \left(\eta_{1}+\eta_{2}\right), \\
\eta_{i}=p_{i}(t) x+q_{i}(t)+\eta_{i 0}, \quad(i=1,2), \\
p_{i}(t)=\frac{\beta_{i}}{a_{0} \int^{t} f(s) d s+b_{0}}, \\
q_{i}(t)=-\int \frac{\left[\beta_{i}^{3}+c_{0} \beta_{i}\right] f(\tau)}{\left[a_{0} \int^{\tau} f(s) d s+b_{0}\right]^{2}} d \tau .
\end{gathered}
$$

To our knowledge, solutions (29) and (39) have not been shown in other literatures.

\section{Conclusions}

In summary, we have presented a method to derive a new auto-BT that consisted of (18) and (19) of (1). The new auto$\mathrm{BT}$ also only involves one quadratic homogeneity equation to be solved. Comparing with auto BT (2), the new auto-BT in this paper does not require the coefficients of the equation to be linearly dependent; in fact, there are no restrictions between these coefficients. The crucial step involves making the assumption that the solution of (1) is of form (4). The key idea of the method is to remove the assumption, which is setting the coefficients of $F^{\prime}, F^{\prime \prime}$, and $F^{\prime \prime \prime}$ to zero, in the homogeneous balance method. It is worthwhile to point out that this method is universal and may be applicable to other nonlinear evolution equations to construct their auto-BT and multisoliton solutions $[12,13]$. Based on the new auto-BT, we obtain a new exact soliton-like solution containing 2-solitary wave under constraint $g(t)=\left[a_{0} \int^{t} f(s) d s+b_{0}\right] f(t)$.

\section{Conflict of Interests}

The author declares that there is no conflict of interests regarding the publication of this paper.

\section{Acknowledgments}

This project is supported by National Natural Science Foundation of China under Grant no. 11371311 and the Natural Science Foundation of the Higher Education Institutions of Jiangsu Province under Grant no. 12KJB110020. 


\section{References}

[1] R. Grimshaw, "Slowly varying solitary waves. I: Korteweg-de Vries equation," Proceedings of the Royal Society. London A: Mathematical and Physical Sciences, vol. 368, no. 1734, pp. 359375, 1979.

[2] N. Nirmala, M. J. Vedan, and B. V. Baby, "Auto-Bäcklund transformation, Lax pairs, and Painlevé property of a variable coefficient Korteweg-de Vries equation. I," Journal of Mathematical Physics, vol. 27, no. 11, pp. 2640-2643, 1986.

[3] W. Hong and Y. D. Jung, "Auto-Bäcklund transformation and analytic solutions for general variable-coefficient KdV equation," Physics Letters A, vol. 257, no. 3-4, pp. 149-152, 1999.

[4] M. Wang and Y. Wang, "A new Bäcklund transformation and multi-soliton solutions to the $\mathrm{KdV}$ equation with general variable coefficients," Physics Letters A, vol. 287, no. 3-4, pp. 211216, 2001.

[5] E. Fan, "Auto-Bäcklund transformation and similarity reductions for general variable coefficient KdV equations," Physics Letters. A, vol. 294, no. 1, pp. 26-30, 2002.

[6] S. Zhang, "Application of Exp-function method to a KdV equation with variable coefficients," Physics Letters A, vol. 365, no. 5-6, pp. 448-453, 2007.

[7] J. Weiss, M. Tabor, and G. Carnevale, “The Painlevé property for partial differential equations," Journal of Mathematical Physics, vol. 24, no. 3, pp. 522-526, 1983.

[8] M. L. Wang, "Solitary wave solutions for variant Boussinesq equations," Physics Letters A, vol. 199, no. 3-4, pp. 169-172, 1995.

[9] M. Wang, "Exact solutions for a compound KdV-Burgers equation," Physics Letters A, vol. 213, no. 5-6, pp. 279-287, 1996.

[10] E. Fan and H. Zhang, "New exact solutions to a system of coupled KdV equations," Physics Letters A, vol. 245, no. 5, pp. 389-392, 1998.

[11] E. Fan, "Two new applications of the homogeneous balance method," Physics Letters A, vol. 265, no. 5-6, pp. 353-357, 2000.

[12] C.-P. Liu and L. Zhou, "A new auto-Bäcklund transformation and two-soliton solution for $(3+1)$-dimensional Jimbo-Miwa equation," Communications in Theoretical Physics, vol. 55, no. 2, pp. 213-216, 2011.

[13] C. Liu, "A modified homogeneous balance method and its applications," Communications in Theoretical Physics, vol. 56, no. 2, pp. 223-227, 2011. 


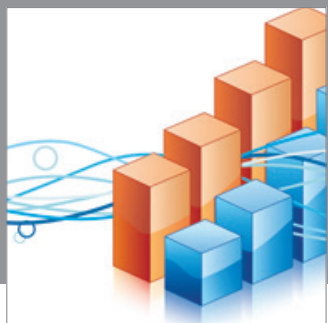

Advances in

Operations Research

mansans

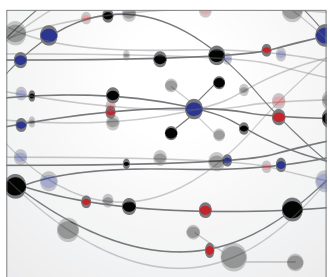

The Scientific World Journal
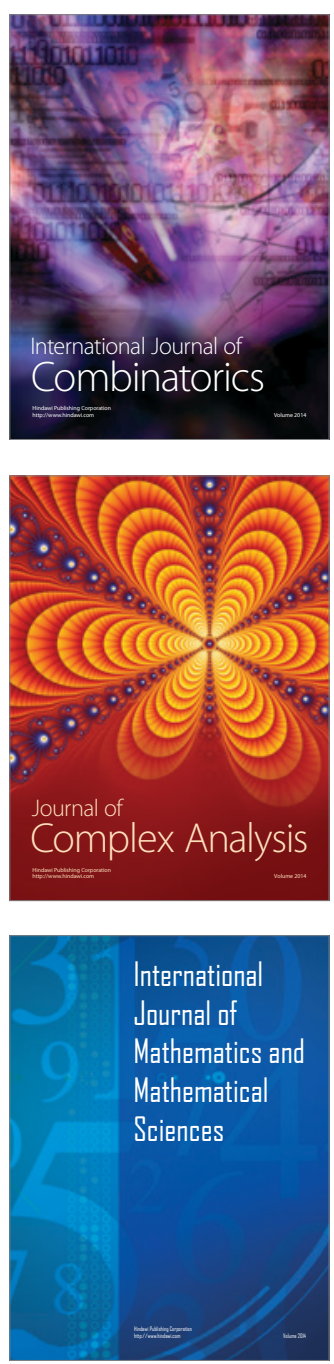
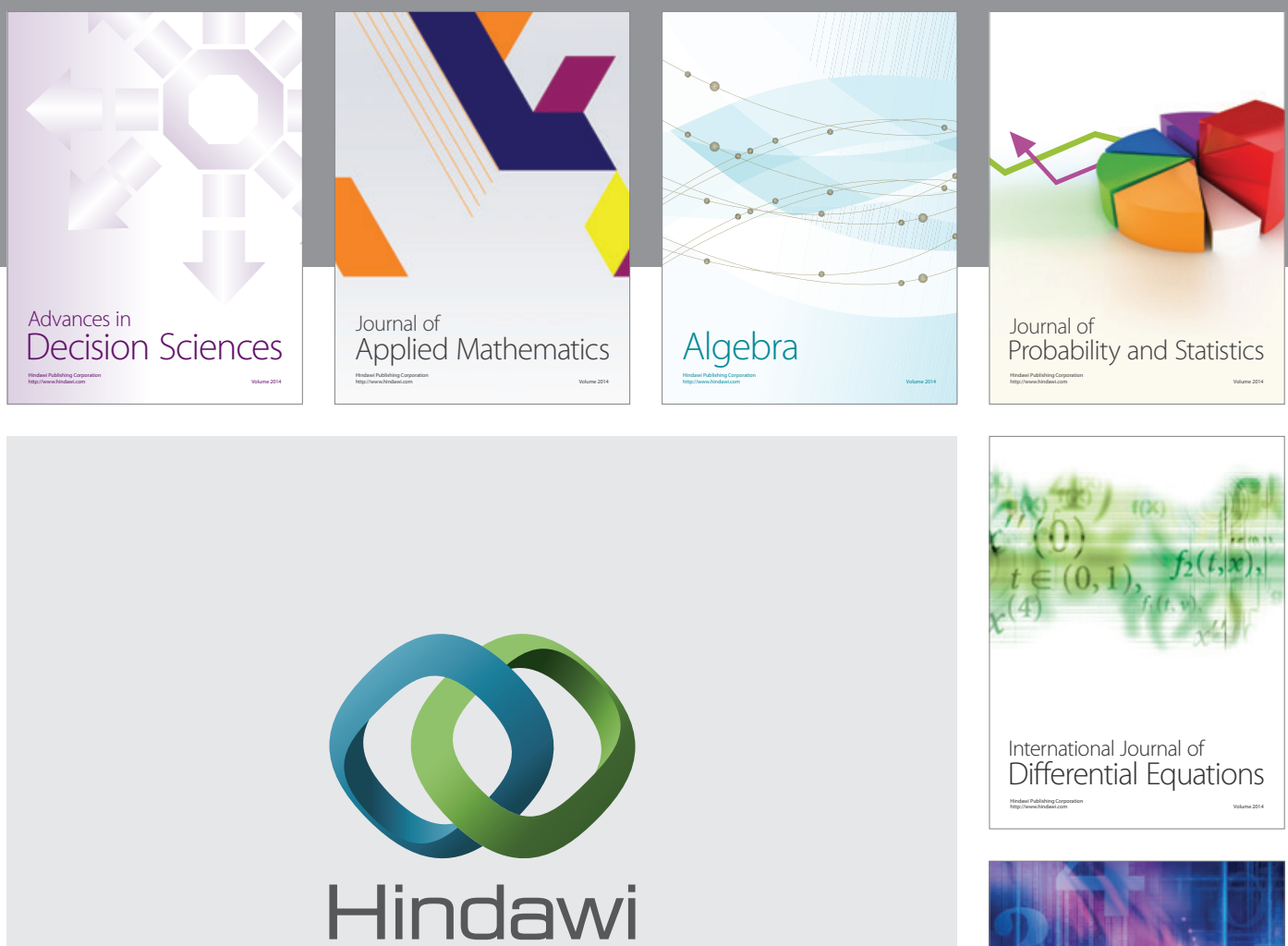

Submit your manuscripts at http://www.hindawi.com
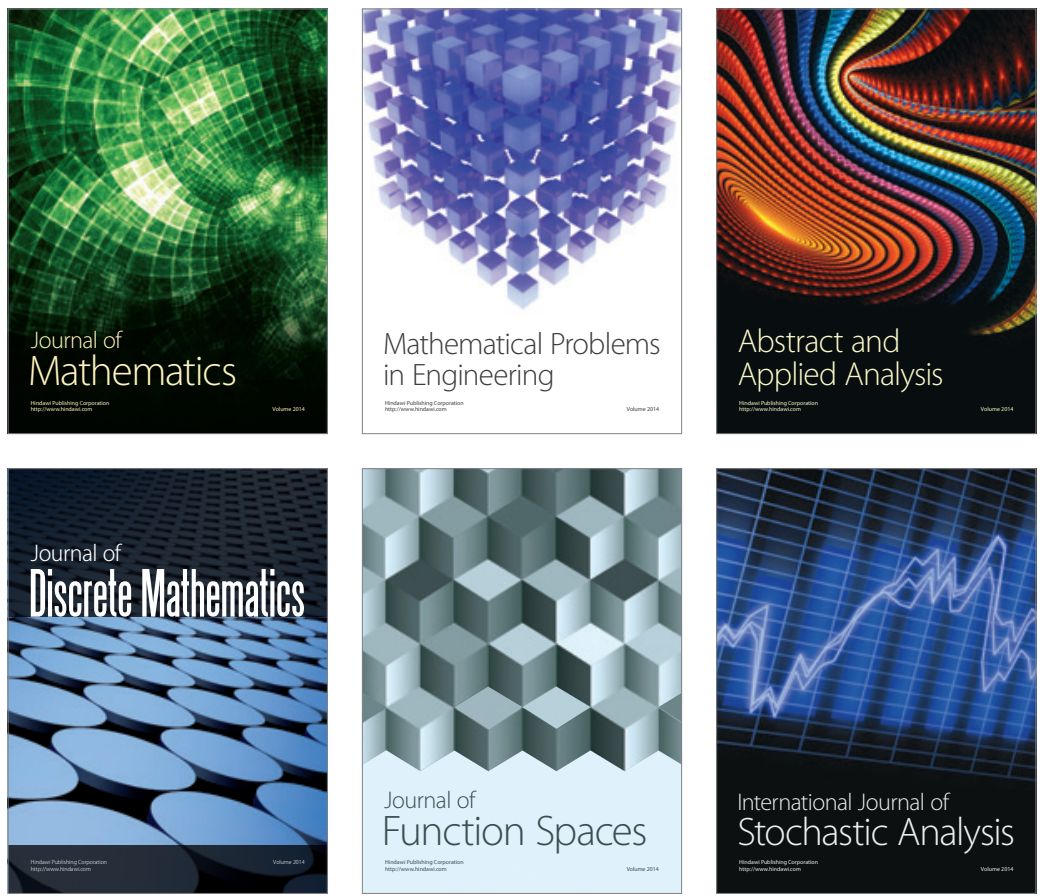

Journal of

Function Spaces

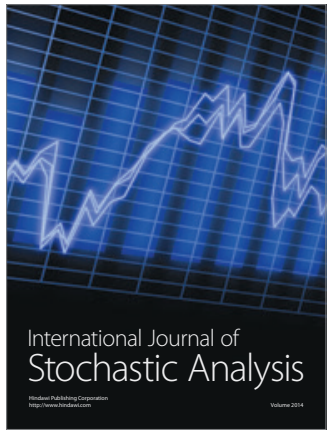

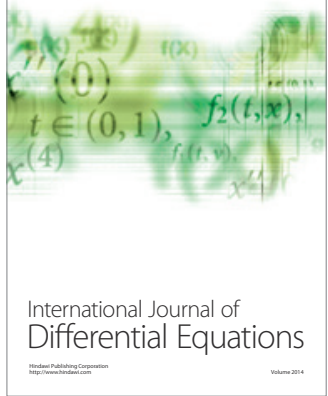
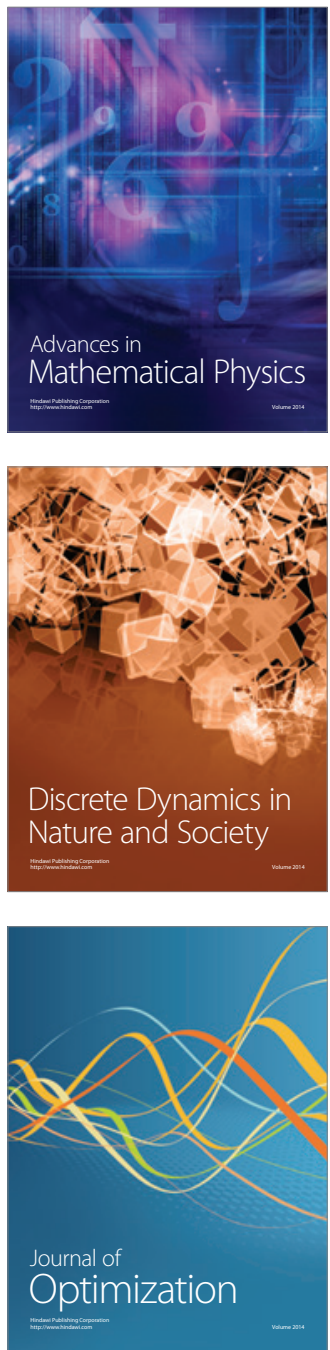